\title{
Spectral Efficiency of the Multiway Massive System over Rician Fading Channels
}

\author{
Junyi He $\mathbb{D}^{\mathbb{D}}$, Junnan Zhang $\mathbb{D}$, Cheng Song $\mathbb{D}$, and Mengxiang $W u$ \\ College of Computer Science and Technology, Henan Polytechnic University, 454000 Jiaozuo, Henan, China \\ Correspondence should be addressed to Junyi He; hejunyi@hpu.edu.cn
}

Received 15 October 2020; Revised 20 December 2020; Accepted 25 January 2021; Published 25 February 2021

Academic Editor: Khaled Maaiuf Rabie

Copyright (c) 2021 Junyi He et al. This is an open access article distributed under the Creative Commons Attribution License, which permits unrestricted use, distribution, and reproduction in any medium, provided the original work is properly cited.

\begin{abstract}
In this study, we consider a multiway massive multi-input multi-output (MIMO) relay network over Rician fading channels, where all users intend to share their information with the other users via amplify-and-forward (AF) relays equipped with a great number of antennas. More practical, the imperfect channel state information (CSI) is taken into account. To evaluate the performance of the considered networks, we derived an analytical approximation expression for the spectral efficiency with zero-forcing (ZF) receivers in a closed form. To obtain more insights, the asymptotic analysis as the number of relay antenna approaching infinity is carried out. Finally, the power scaling law is analyzed for two scenarios. The results reveal that (1) massive MIMO is capable of compensating the loss caused by Rician fading, (2) the sum spectral efficiency increases with the increase of the Rician factor, and (3) deploying large-scale antenna is effective to save cost and keep performance.
\end{abstract}

\section{Introduction}

Massive multiple-input multiple-output in mobile communication technology networks has greatly improved the fluency and stability of communication and provides users with a better experience, which has been defined as a core technology of the fifth generation mobile $[1,2]$. Massive MIMO technology is first setup by Marzetta [3] who releases the multicell multiuser noncooperative system, and now, it has got attention on both industry and academic. By deploying tens or hundreds of antennas at the base station (BS) and servicing many users, massive MIMO has an advantage capability to increase the spectral efficiency and energy efficiency by orders of magnitude [4] and, to some extent, preclude the interuser interference (IUI) with low-complexity linear precoding/detecting schemes such as maximal-ratio combining (MRC), maximal-ratio transmission (MRT), zero-forcing, and minimum mean square error (MMSE) $[5,6]$.

At the same time, multiway relay networks have a significant role on practical application scenarios, e.g., data transmission in multimedia conference call and exchanging information occurring in sensor nodes and data centres in wireless communication $[7,8]$. Thanks to the multiplexing gain, multiway relay networks have become an upgrading technology to further push the performance of spectral efficiency of relay networks $[9,10]$.

In order to obtain the advantages of massive MIMO and multiple relays, the combination of the two technologies has sparked a great interest [11-15]. In [11], the authors investigated the performance of the multiway relay networks, which figures out the transmit power of each user and relay stays in an increasing trend as the number of relay antennas decreases. Moreover, authors in [12] derive multiway massive MIMO relay networks in wireless communication and the technology of power transfer at the same time. It is shown in [13] that the transmit powers at user and relay nodes can be reduced with large antenna array and keep a fixed quality of service in the multiway massive MIMO networks. In [14], the authors study the performance of multiway relay networks and release that the pairwise multiway relay network achieves the maximum multiplexing gain whenever the number of participating sources is limited to two. In [15], the asymptotic performance of MWRNs with massive MIMO by modelling the channels is investigated, and it concludes that the transmitting power of the user node 
is inversely proportional to the number of antennas when the channel is aging. However, the common of the above works has the assumption that all communication are accomplished under the conditions of Rayleigh fading channels [16], which is not practical in some scenarios of line of sights (LoS) environments, such as television broadcasting, satellite communication, and radio relay communication $[17,18]$.

Motivated by the above discussion, we consider a multiway massive MIMO relay network over Rician fading channels with ZF processing in this study. More practical, we suppose that the channel is not perfect, which can be estimated by the massive MIMO relay with MMSE. Specifically, by utilizing the ZFR/ZFT algorithm, we obtain an approximate analytical evaluation for the spectral efficiency of considered multiway massive MIMO relay networks in close. To obtain more insights, the power scaling law is explored as the amount of relay antennas grows to infinity. The main contributions of the study are shown as follows:

(1) With fixing the value of the Rician factor and comparing the trend of the sum spectral efficiency, we find out that massive MIMO can compensate the loss caused by Rician fading;

(2) The sum spectral efficiency grows logarithmic when the Rician fading factor $K$ grows, and moreover, the spectral efficiency presents highlight when $K=0$;

(3) Using the estimated amount obtained, the closed form for the spectral efficiency with ZF processing is obtained. Furthermore, the power scale laws are involved in two parts. And in the first case, $M \longrightarrow \infty$; we find that the spectral efficiency increases as the antennas increases. As for the other case that the transmit power of users reduces with $M$ growing, the effects of estimate error and interpair interference can be ignored.

The rest of this study is organized as follows: in Section 2, we introduce the network model and give the channel model, channel estimation, and information transmission. In Section 3, we analyze the spectral efficiency. The power scaling law is considered in Section 4, and the numerical results are shown in Section 5. The last section concludes the study.

Notations: superscript $(A)^{T}$ is the transpose of the matrix $A,(A)^{*}$ is the conjugate of the matrix, and $(A)^{H}$ represents the Hermitian of the matrix A. While, $\mathbb{E}\{|*|\}$ and $\operatorname{Var}\{|*|\}$ represent the expectation and the variance operators, respectively. $A_{k}$ and $a_{k}$ are the $k^{\text {th }}$ columns of matrix A. The notation $\mathrm{A} \sim \mathrm{CN}(0,1)$ denotes that $\mathrm{A}$ is a circularly symmetric Gaussian distributed random variable.

\section{Network Model}

We consider a multiway massive MIMO AF relay network as shown in Figure 1, which includes one $M$ antenna relay and $N$ single antenna users. Each user with one antenna tries to exchange their information with the other $N-1$ users with the aid of the AF relay station, which is equipped with $M$ antennas $(M>>\mathrm{N})$. We suppose that the direct link cannot

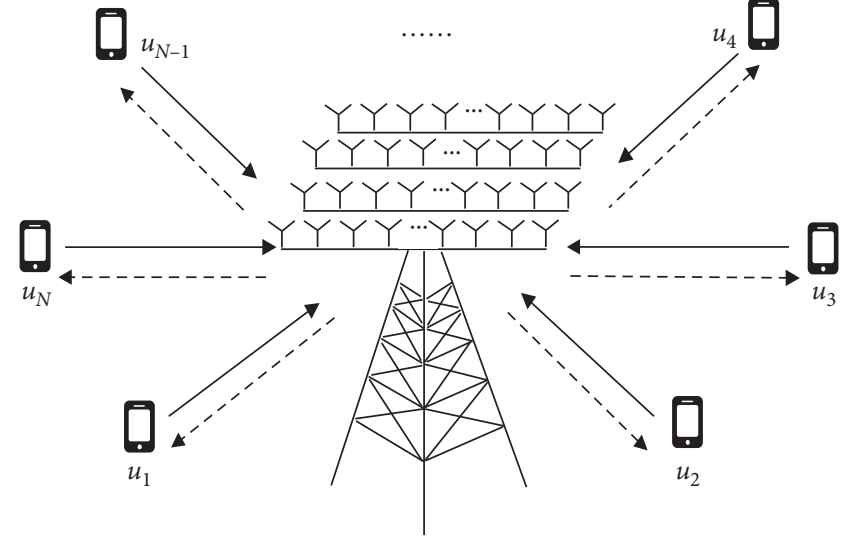

Figure 1: System model.

be achieved caused by high path loss and/or severe shadowing.

2.1. Channels Model. Let $G \in \mathbb{C}^{M \times N}$ be the channel matrix from the $N$ users to the relay, which contains the small-scale and/or the large-scale fading, and it can be written as

$$
G=\mathrm{HD}^{(1 / 2)}
$$

where $D \in \mathbb{C}^{N \times N}$ is a diagonal matrix which contains large-scale fading coefficients, with the restrain by $\beta_{n}$ in the $N^{\text {th }}$ diagonal element. Contrast to [19], due to the channel is in Rician fading, it can be written as [20]

$$
H=\sqrt{\frac{K_{n}}{K_{n}+1}} \bar{H}+\sqrt{\frac{1}{K_{n}+1}} \tilde{H}, \quad X \in\{A, B\} .
$$

In the above formula, $K_{n}$ is the Rician $K$-factor coefficient which combines the $N-1$ user pair and the relay, while $\widetilde{H}$ is the random part of the Rician fading channels with the communication of the $N-1$ user to relay, and all elements in both are independent and identically distributed (i.i.d.) $\mathrm{CN}(0,1)$ random variables $[21] . \bar{H}$ is the deterministic part of Rician fading channels with the restrain of the $N-1$ user pair, which is an arbitrary rank $[\bar{H}]_{m n}=e^{-j(m-1)(2 \pi d / \lambda) \sin \left(\theta_{n}\right)}$, where $\lambda$ is the wavelength, $d$ is the antenna spacing, and $\theta_{n}$ stands for the arrival angle of the $N^{\text {th }}$ user.

Under the time division duplex (TDD) operations, the transmission protocol can be described when the information exchange is totally finished among all $N$ users. When using TDD operation, there are three parts in each coherence interval: channel estimation, multiple access phase, and broadcasting phase.

2.2. Channel Estimation. In a wireless communication system, it is a challenge to obtain perfect CSI, especially in massive MIMO systems which equipped with the large number of antenna array at BS. As in [21], we assume that the deterministic component and Rician factor are known during transmission. We define that $T$ is the length of coherent interval, and $\tau$ stands for the training duration in each coherent interval, and set $T>\tau$. In the training phase, we 
assume that all users send their pilot sequences to the relay at the same time, where the pilot sequences are mutually orthogonal. In this situation, we require $\tau \geq \mathrm{N}$, Then, $\widehat{G}$ can be written as [19]

$$
\widehat{G}=\bar{G}\left[\Omega\left(\Omega+I_{N}\right)^{-1}\right]^{(1 / 2)}+\widehat{G}_{w}\left[\left(\Omega+I_{N}\right)^{-1}\right]^{(1 / 2)},
$$

where $\bar{G}$ is the deterministic component with $\bar{G}=\bar{H} D^{(1 / 2)}$, and $\widehat{G}_{w}$ is the estimate channel of random component.

During the training step, orthogonal pilot sequences realize the matrix $\sqrt{p_{p}} \Phi \in \mathbb{C}^{\tau \times N}(\tau \geq N)$, constraint with $F^{H} F=I_{N}$, where $F \triangleq \Phi\left[\Omega\left(\Omega+I_{N}\right)^{-1}\right]^{(1 / 2)}$, and $p_{p}=\tau p_{u}$ is the transmitted pilot power. The pilot matrix with noise received by the base station can be represented as

$$
Y_{p}=\sqrt{p_{p}} G \Phi^{T}+N
$$

where $N \sim \mathrm{CN}\left(0, I_{M}\right)$ is the AWGN vector at the relay. And the remaining term of the receive matrix is

$$
Y_{p, w}=\sqrt{p_{p}} G_{w}\left[\left(\Omega+I_{N}\right)^{-1}\right]^{(1 / 2)} \Phi^{T}+N .
$$

Then, using MMSE, we can get the estimate of random component $G_{w}$ as follows:

$$
\widehat{G}_{w}=\frac{1}{\sqrt{p_{p}}} Y_{p} F^{*} D,
$$

where $D \triangleq\left(\left(1 / p_{p}\right) D^{-1}+I_{N}\right)$.

With the formula (5), we have

$$
\widehat{G}_{w}=\left(G_{w}+\frac{1}{p_{p}} Z\right) D,
$$

where $Z \triangleq \mathrm{NF}^{*}, Z \sim \mathrm{CN}\left(0, I_{M}\right)$ is the AWGN vector, and $\widehat{G}_{w} \sim \mathrm{CN}\left(0, \widehat{D}_{w}\right)$, and $\left[\widehat{D}_{w}\right]_{n n}=\left(\tau P_{p} \beta_{n}^{2} / \tau P_{p} \beta_{n}+1\right)$.

Substituting (7) to (3), the channel estimate matrix of $G$ can be obtained as [22]

$$
\widehat{G}=G-E,
$$

where $\widehat{G}$ and $E$ are independent with each other, and $E$ presents the estimation error matrix. In addition, $\widehat{G} \sim \mathrm{CN}(0, \widehat{D})$ and $E \sim \mathrm{CN}\left(0, D_{E}\right)$, where $\widehat{D}$ and $\mathrm{D}_{\mathrm{E}}$ are the diagonal matrices, and its diagonal elements are $[\widehat{D}]_{n n}=$ $\sigma_{n}^{2}=\left(K_{n} / K_{n}+1\right) \sqrt{\left(\tau P_{p} \beta_{n}^{2} / \tau P_{p} \beta_{n}+1\right)}+\left(\tau P_{p} \beta_{n}^{2} / \tau P_{p} \beta_{n}+1\right)$ $\sqrt{1 /\left(K_{n}+1\right)}$ and $\left[D_{E}\right]_{n n}=\sigma_{e, n}^{2}=\beta_{n}-\sigma_{n}^{2}$, respectively.

\subsection{Information Transmission}

2.3.1. Multiple Access Phase. Based on the estimated channels, data are sent to the relay simultaneously by all users. Let $x_{k}$ be the transmitted signal from $k^{\text {th }}$ user with $\mathbb{E}\left\{\left|x_{k}\right|^{2}\right\}=1$. Thus, at the relay, the received signal is

$$
y_{R}=\sqrt{P_{u}} G x+n_{r} .
$$

In above formula, $x \triangleq\left[x_{1}, \ldots, x_{K}\right]^{T}$, and $n_{r} \sim \mathrm{CN}\left(0, I_{m}\right)$ indicates the AWGN vector at the relay. With the aid of the estimated channel and $\mathrm{ZF}$ processing technique, the relay combines the received signals from all $M$ antennas as

$$
\tilde{y}_{R}=W^{T} y_{R}
$$

where $W^{T}$ is the ZF receiver and can be obtained from [23]

$$
W^{T}=\left(\widehat{G}^{H} \widehat{G}\right)^{-1} \widehat{G}^{H} \text {. }
$$

2.3.2. Broadcasting Phase. In this state, it takes $K-1$ time slots to send signal to all users with the help of the relay. The relay tries to send $x_{k+1}$ to user $k$, when the $t^{\text {th }}$ time slot comes. Thus, at the relay, for the $t^{\text {th }}$ time slots, the transmitted signal vector can be written as [9]

$$
S_{R}^{(t)}=\sqrt{\alpha^{(t)} P_{u}} B^{(t)} x+\sqrt{\alpha^{(t)}} C^{(t)} n_{r}
$$

where $B^{(t)} \triangleq A \Pi^{(t)} W^{T} G$ and $C^{(t)} \triangleq A \Pi^{(t)} W^{T}$, and $\alpha^{(t)}$ is chosen to satisfy the power constraint at the relay. And $\prod^{(t)} \in \mathbb{C}^{N \times N}$ is the permutation matrix at the $t^{\text {th }}$ time slot as in [8]. A, which represents the ZF precoding matrix, can be written as

$$
\begin{aligned}
A & =\widehat{G}^{*}\left(\widehat{G}^{T} \widehat{G}^{*}\right)^{-1}, \\
\mathbb{E}\left\{\left\|S_{R}^{(t)}\right\|^{2}\right\} & =P_{r} .
\end{aligned}
$$

Substituting (12) into (14), we can have

$$
\alpha^{(t)}=\frac{P_{r}}{P_{u} Q_{1}^{(t)}+P_{u} Q_{2}^{(t)}+Q_{3}^{(t)}},
$$

where

$$
\begin{aligned}
& Q_{1}^{(t)} \triangleq \mathbb{E}\left\{\operatorname{Tr}\left[\left(A \Pi^{(t)} W^{T} \widehat{G}\right)\left(A \Pi^{(t)} W^{T} \widehat{G}\right)^{H}\right]\right\}, \\
& Q_{1}^{(t)} \triangleq \mathbb{E}\left\{\operatorname{Tr}\left[\left(A \Pi^{(t)} W^{T} E\right)\left(A \Pi^{(t)} W^{T} E\right)^{H}\right]\right\}, \\
& Q_{1}^{(t)} \triangleq \mathbb{E}\left\{\operatorname{Tr}\left[\left(A \Pi^{(t)} W^{T}\right)\left(A \Pi^{(t)} W^{T}\right)^{H}\right]\right\} .
\end{aligned}
$$

From (12), the transmitted signal can be obtained, and the $\mathrm{K}$ users meet

$$
y_{u}^{(t)}=G^{T} S_{R}^{(t)}+n_{u}^{(t)}=\sqrt{\alpha^{(t)} P_{u}} G^{T} B^{(t)} x+\sqrt{\alpha^{(t)}} G^{T} C^{(t)} n_{r}+n_{u}^{(t)},
$$

where $n_{u} \sim \mathrm{CN}\left(0, I_{N}\right)$ is the AWGN vector in the end.

\section{Spectral Efficiency Analysis}

In this section, we derive a closed-form expression for the spectral efficiency of massive MIMO multirelay systems over Rician fading channels. In terms of the other time slots, the same analysis can meet the requirement. Depending on the effort of [24], the received signal at the $n^{\text {th }}$ user $y_{u, n}^{(1)}$ can be shown as 


$$
y_{u, n}^{(1)}=\sqrt{\alpha^{(1)} P_{u}} \mathbb{E}\left\{g_{n}^{T} b_{n+1}^{(1)}\right\} x_{n+1}+\tilde{N}_{n}^{(1)},
$$

where

$$
\begin{aligned}
\tilde{N}_{n}^{(1)} \triangleq & \sqrt{\alpha^{(1)} P_{u}}\left(g_{n}^{T} b_{n+1}^{(1)}-\mathbb{E}\left\{g_{n}^{T} b_{n+1}^{(1)}\right\} x_{n+1}\right) \\
& +\sqrt{\alpha^{(1)} P_{u}} \sum_{\substack{i=1 \\
i(n+1)}}^{N} g_{n}^{T} b_{i}^{(1)} x_{i}+\sqrt{\alpha^{(1)}} g_{n}^{T} C^{(1)} n_{r}+n_{u, n}^{(1)}
\end{aligned}
$$

For the $k^{\text {th }}$ user, the worst-case Gaussian noise will produce reachable spectral efficiency, and the formula is

$$
\mathrm{SE}_{n}^{(1)}=\left(\frac{T-\tau}{T}\right)\left(\frac{N-1}{N}\right) \log _{2}\left(1+\frac{\alpha^{(1)} P_{u}\left|\mathbb{E}\left\{g_{n}^{T} b_{n+1}^{(1)}\right\}\right|^{2}}{\operatorname{Var}\left(\tilde{N}_{n}^{(1)}\right)}\right) \text {. }
$$

To obtain the closed form of the spectral efficiency, we need to calculate $\mathbb{E}\left\{g_{n}^{T} b_{n+1}^{(1)}\right\}$ and $\operatorname{Var}\left(\tilde{N}_{n}^{(1)}\right)$. Due to the independence relationship of $\widehat{G}$ and $E$, we can obtain

$$
\mathbb{E}\left\{g_{n}^{T} b_{n+1}^{(1)}\right\}=\mathbb{E}\left\{\hat{g}_{n}^{T} A \Pi^{(1)} W^{T} \widehat{g}_{n+1}\right\}=1 .
$$

Because $\tilde{N}_{n}^{(1)}$ has a complex form, which cannot be calculated, and also, it is difficult to get the exact form of $\operatorname{Var}\left(\tilde{N}_{n}^{(1)}\right)$. However, by using the law of Large Numbers [9], we can have the following approximation.

Theorem 1. With $M \longrightarrow \infty$, the spectral efficiency can be evaluated as

$$
\mathrm{SE}_{n}^{(1)} \longrightarrow\left(\frac{T-\tau}{T}\right)\left(\frac{N-1}{N}\right) \times \log _{2}\left(1+\frac{\alpha^{(1)} P_{u}}{\alpha^{(1)} P_{u} \sum_{i=1}^{N} I_{n, i}+\alpha^{(1)} \zeta_{n}+1}\right)
$$

where

$$
\begin{aligned}
& \alpha^{(1)} \triangleq \frac{M(M-N) P_{r}}{\operatorname{MP}_{u} \sum_{n=1}^{N}\left(1 / \sigma_{n}^{2}\right)+P_{u} \sum_{n=1}^{N} \sigma_{e, n}^{2} \sigma+\sigma}, \\
& I_{n, i} \triangleq \frac{M \sigma_{i-1}^{2} \sigma_{e, i}^{2}+M \sigma_{n+1}^{2} \sigma_{e, k}^{2}+\sigma_{n+1}^{2} \sigma_{i-1}^{2} \sigma_{e, k}^{2} \sigma_{e, i}^{2} \sigma}{M(M-N) \sigma_{i-1}^{2} \sigma_{n+1}^{2}}, \\
& \zeta_{n} \triangleq \frac{M+\sigma_{n+1}^{2} \sigma_{e, k}^{2} \sigma}{M(M-N) \sigma_{n+1}^{2}}, \\
& \sigma \triangleq \sum_{n^{\prime}=1}^{N} \frac{1}{\sigma_{n^{\prime}}^{2} \sigma_{n^{\prime}+1}^{2}} .
\end{aligned}
$$

Proof. See Appendix.

\section{Power Scaling Laws}

In this part, the power scaling law is studied for the considered networks. We mainly focus on these two cases: (1) fix
$P_{u}, P_{r}$, and set $p_{p}=\left(E_{p} / M^{\alpha_{p}}\right)$ with the restrain of $\alpha_{p}>0 ;(2)$ fix $E_{u}, E_{r}$, and $P_{p}$, and set $p_{u}=\left(E_{u} / M^{\alpha_{u}}\right)$ and $p_{r}=\left(E_{r} / M^{\alpha_{r}}\right)$, with the restrain of $\alpha_{u}>0, \alpha_{r}>0$.

First case: the power scaling laws can be calculated as below when $M$ has a trend to infinity.

Theorem 2. Fix $P_{u}, P_{r}$, and $E_{p}$; set $p_{p}=\left(E_{p} / M^{\alpha}{ }^{\alpha}\right)$, with the restrain of $\alpha_{p}$, we have

$$
\begin{aligned}
& \mathrm{SE}_{n}^{(1)} \longrightarrow\left(\frac{T-\tau}{T}\right)\left(\frac{N-1}{N}\right) \\
& \quad \times \log _{2}\left(1+\frac{\alpha^{(1)} P_{u}}{\widehat{\alpha}^{(1)} P_{u} \sum_{i=1}^{N} \widehat{I}_{n, i}+\widehat{\alpha}^{(1)} \widehat{\zeta}_{n}+1}\right),
\end{aligned}
$$

where

$$
\begin{aligned}
\widehat{\alpha}^{(1)} \triangleq \frac{M(M-N) P_{r}}{M P_{u} \sum_{n=1}^{N}\left(1 / \sigma_{n}^{2}\right)+P_{u} \sum_{n=1}^{N} \sigma_{e, n}^{2} \sigma+\sigma}, \\
\widehat{I}_{n, i} \triangleq \frac{M \sigma_{i-1}^{2} \sigma_{e, i}^{2}+M \sigma_{n+1}^{2} \sigma_{e, k}^{2}+\sigma_{n+1}^{2} \sigma_{i-1}^{2} \sigma_{e, k}^{2} \sigma_{e, i}^{2} \sigma}{M(M-N) \sigma_{i-1}^{2} \sigma_{n+1}^{2}} \\
\widehat{\zeta}_{n} \triangleq \frac{M+\sigma_{n+1}^{2} \sigma_{e, k}^{2} \sigma}{M(M-N) \sigma_{n+1}^{2}}, \\
\sigma^{2} \triangleq \frac{K_{n}}{K_{n}+1} \sqrt{\frac{\tau E_{p} \beta_{n}^{2}}{\tau E_{p} \beta_{n}+M^{\alpha_{p}}}+\frac{\tau E_{p} \beta_{n}^{2}}{\tau E_{p} \beta_{n}+M^{\alpha_{p}}} \sqrt{\frac{1}{K_{n}+1}} .}
\end{aligned}
$$

Proof. By substituting $P_{p}=\left(E_{p} / M^{\alpha}\right)$ and making $M$ into infinity.

Note 1: Theorem 2 shows that the sum spectral efficiency is not relevant to $\alpha_{p}$, due to the reason that the estimation error becomes a regular number when $P_{p} \longrightarrow 0$. Furthermore, with the antennas having a trend of growth, the spectral efficiency increases.

Second case: the users transmit power reduces when the number of antenna $M$ increases.

Theorem 3. Fix $E_{u}, E_{r}$, and $P_{p}$, when $P_{u}=\left(E_{u} / M^{\alpha_{u}}\right)$ and $P_{r}=\left(E_{r} / M^{\alpha_{r}}\right)$, with the restraint $\alpha_{u}>0, \alpha_{r}>0$.

Proof. By substituting $P_{u}=\left(E_{u} / M^{\alpha_{u}}\right), P_{r}=\left(E_{r} / M^{\alpha_{r}}\right)$ and taking $M$ into infinity.

Note 2: it is shown that the effects of estimate error and interpair interference can be ignored when $M$ climbs to infinity. When meeting the condition that $0<\alpha_{u}, \alpha_{r}<1$, the spectral efficiency of the user grows in an unlimited trend. When $\alpha_{u}=\alpha_{r}=1$, the spectral efficiency of the user is fixed. When $\alpha_{u}>1, \alpha_{r}>1$, the spectral efficiency drops to zero as $M \longrightarrow \infty$. 


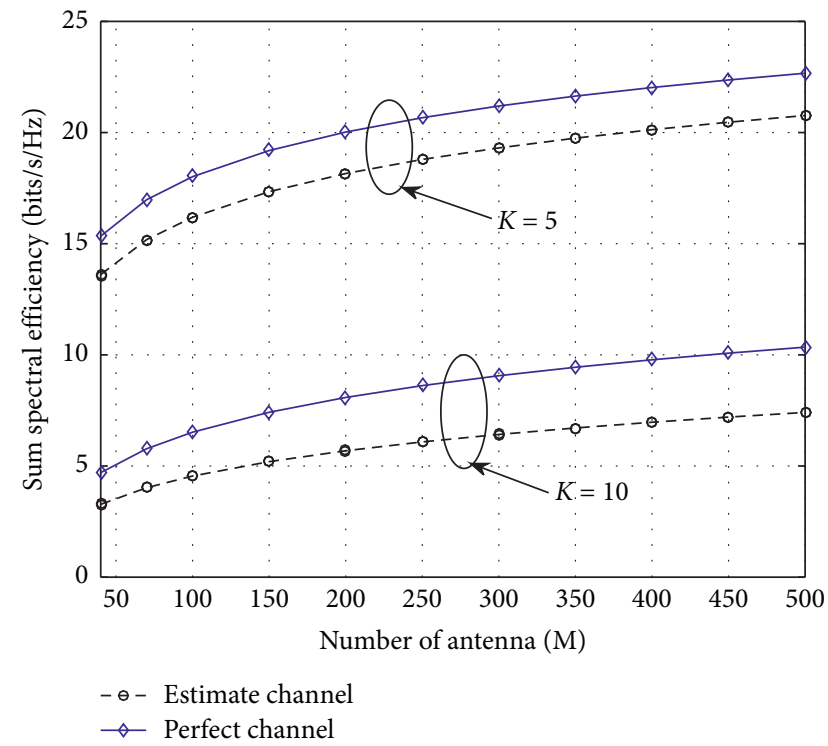

Figure 2: Sum spectral efficiency via number of antennas with $K=10,5$.

\section{Numerical Results}

The sum spectral efficiency is taken as our performance indictor. The sum spectral efficiency can be rewritten follows:

$$
\mathrm{SE}_{\text {sum }}=\sum_{n=1}^{N} \mathrm{SE}_{n}^{(1)} \mathrm{bit} / \mathrm{s} / \mathrm{Hz}
$$

Figure 2 shows the sum spectral efficiency in a multiway massive MIMO system with ZF processing versus number of antennas with different values of $K$. The diamond dotted represents the Monte Carlo results of the spectral efficiency of the multichannel massive MIMO relay, while the dotted circle line represents the analysis result of Part 3. In this figure, we fix $P_{u}=10 \mathrm{~dB}, N=8, P_{p}=P_{u}$, and $P_{r}=2 \mathrm{NP}_{u}$. In this figure, we can find that the simulation value is in agreement with the Monte Carlo result. Furthermore, we can easily conclude that the $\mathrm{ZF}$ processing has a slow growth when the antenna increases heavily.

Figure 3 shows the sum spectral efficiency of the considered system with ZF versus SNR when $K$ is set to 10 and 5 . As can be seen from Figure 3, spectral efficiency grows larger with the increase of SNR. We can learn that the ZF processing has a better performance with SNR growing in a multiway massive MIMO system. In addition, the spectral efficiency decreases with $K$ growing, which means the performance of the considered system is limited by the Rician factor.

Figure 4 investigates the sum spectral efficiency with different values of $K$. As shown in figure, with the increase of $K$, the sum spectral efficiency can get an apparent decline,

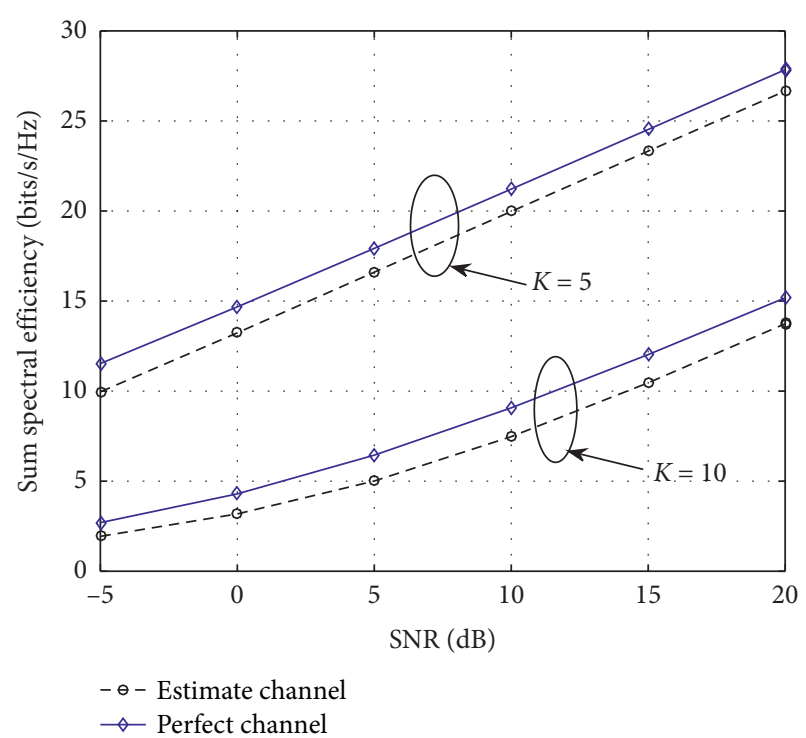

FIgURE 3: Sum spectral efficiency via SNR with $K=10,5$.

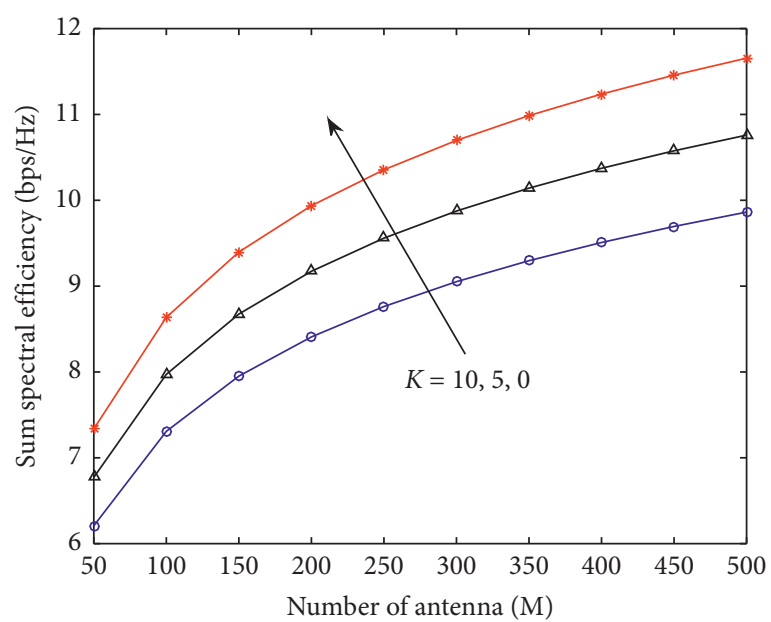

FIgURE 4: Sum spectral efficiency via the Rician fading factor.

which is going to say, the sum spectral efficiency descends with the Rician factor $K$ rising.

Figure 5 shows the power scaling law versus $M$ for the transmit power of each user $P_{u}=\left(E_{u} / M^{\alpha_{u}}\right)$ and the relay $p_{r}=$ $\left(E_{r} / M^{\alpha_{r}}\right)$ of the considered system. In this figure, we set $N=8$, $E_{u}=10 \mathrm{~dB}, E_{r}=20 \mathrm{~dB}, P_{p}=10 \mathrm{~d} B, K=10 \mathrm{~dB}$, and $T=200$. As we can see that when $\alpha_{u}, \alpha_{r}>1$, the sum spectral efficiency reduces to zero, which can get that when the transmit power is reduced too much on each user and relay, the sum spectral efficiency will be greatly reduced. When $\alpha_{u}, \alpha_{r}<1$, the sum spectral efficiency grows with the number of antenna $M$ increasing. When $\alpha_{u}=\alpha_{r}=1$, the sum spectral efficiency becomes a constant, which shows that we cannot promote the sum spectral efficiency by making the number of antenna increase. 


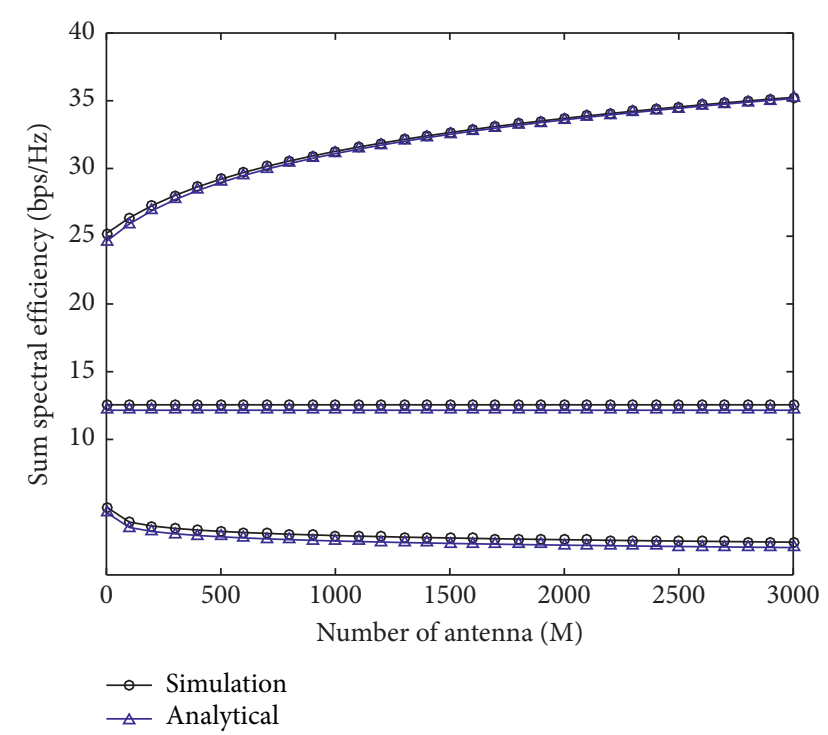

Figure 5: Sum spectral efficiency via $M$.

\section{Conclusions}

In this study, we summarized the influence of the Rician factor on the performance of the multiway massive MIMO relay network with $\mathrm{ZF}$ processing. We deduced an approximate expression for the spectral efficiency in a closed form. Also, the relationship between the Rician factor and the sum spectral efficiency is obtained. And massive MIMO have an ability to compensate the loss caused by Rician fading. Moreover, we show that ZF processing offers a higher spectral efficiency in most circumstances.

\section{Appendix}

1

Lemma 1. Let $X \in \mathbb{C}^{M \times N}, M>N$. Each row of $X$ is $C N(0, D)$, where $D$ is a diagonal matrix. Furthermore, let $\widehat{D} \in \mathbb{C}^{N \times N}$ be another diagonal matrix. Then, we have

$$
\mathbb{E}\left\{\operatorname{Tr}\left[\widehat{D}\left(X^{H} X\right)^{-1}\right]\right\}=\frac{1}{M-N} \sum_{n=1}^{N} \frac{[\widehat{D}]_{n n}}{[D]_{n n}}
$$

Proof. By expressing $\operatorname{Tr}\left[\widehat{D}\left(X^{H} X\right)^{-1}\right]=\sum_{n=1}^{N}(\widehat{[D}]_{n n} /$ $\left.[D]_{n n}\right)\left[W^{-1}\right]_{N N}$, where $W$ is a $K \times K$ central Wishart matrix of $M$ degrees of freedom, and using [10], Lemma 2.10 is obtained.

2

Lemma 2. Let $A \in \mathbb{C}^{M \times M}$, and $X \sim C N\left(0, I_{M}\right)$. Then,

$$
\mathbb{E}\left\{\left|X^{T} A X\right|^{2}\right\}=\operatorname{Tr}\left(A A^{H}\right)+\operatorname{Tr}\left(A A^{*}\right) .
$$

3

Proof of Theorem 1
(1) Derivation of $\alpha^{(1)}$ : from (10), to compute $\alpha^{(1)}$, we need to compute $Q_{1}^{(1)}, Q_{2}^{(1)}$, and $Q_{3}^{(1)}$. Substituting (5) and (7) into (11) yields

$$
Q_{1}^{(1)}=\mathbb{E}\left\{\operatorname{Tr}\left(\widehat{G}^{T} \widehat{G}^{*}\right)^{-1}\right\}=\frac{1}{M-N} \sum_{n=1}^{N}\left(1 / \sigma_{n}^{2}\right) \text {. }
$$

Where in the last equality, we have used Lemma 1. To compute $Q_{2}^{(1)}$, we substitute (5) and (7) into (12) to obtain

$$
\left.Q_{2}^{(1)}=\sum_{n=1}^{N} \sigma_{e, n}^{2} \mathbb{E}\left\{\operatorname{Tr}\left[\Pi^{(1)} \widehat{G}^{H} \widehat{G}^{*}\right)^{-1}\left(\Pi^{(1)}\right)^{H}\left(\widehat{G}^{T} \widehat{G}^{*}\right)^{-1}\right]\right\} \text {. }
$$

From the law of large numbers, we have that $\widehat{G}^{H} \widehat{G} \longrightarrow M \widehat{D}$, and hence, $Q_{2}^{(1)}$ can be approximated as

$$
\begin{aligned}
Q_{2}^{(1)} & \left.\longrightarrow \sum_{n=1}^{N} \sigma_{e, n}^{2} \mathbb{E}\left\{\operatorname{Tr}\left[\Pi^{(1)} \widehat{G}^{H} \widehat{G}^{*}\right)^{-1}\left(\Pi^{(1)}\right)^{H}\left(\widehat{G}^{T} \widehat{G}^{*}\right)^{-1}\right]\right\} \\
& =\frac{\varrho}{M(M-N)} \sum_{n=1}^{N} \sigma_{e, n}^{2} .
\end{aligned}
$$

Where again, we have used Lemma 1 to obtain the last equality.

Similarly, we obtain

$$
Q_{3}^{(1)} \longrightarrow \frac{\varrho}{M(M-N)}
$$

Substituting (A.4), (A.5), and (A.6) into (16), we obtain (24).

(2) Derivation of $\operatorname{Var}\left(\widetilde{N}_{n}^{(1)}\right)$ : from (20), we have

$$
\begin{aligned}
\operatorname{Var}\left(\tilde{N}_{n}^{(1)}\right)= & \alpha^{(1)} P_{u} \operatorname{Var}\left(g_{n}^{T} b_{n+1}^{(1)}\right)+\alpha^{(1)} P_{u} \mathbb{E}\left\{\left|g_{n}^{T} b_{n}^{(1)}\right|^{2}\right\} \\
& +\alpha^{(1)} P_{u} \sum_{\substack{i=1 \\
i \neq(n, n+1)}}^{N} \mathbb{E}\left\{\left|g_{n}^{T} b_{i}^{(1)}\right|^{2}\right\} \\
& +\alpha^{(1)} \mathbb{E}\left\{\left\|g_{n}^{T} C^{(1)}\right\|^{2}\right\}+1 .
\end{aligned}
$$

(a) Compute $\operatorname{Var}\left(g_{n}^{T} b_{n+1}^{(1)}\right)$, we have

$$
\operatorname{Var}\left(g_{n}^{T} b_{n+1}^{(1)}\right)=V_{1}+V_{2}+V_{3} \text {. }
$$

Where

$$
\begin{aligned}
& V_{1} \triangleq \mathbb{E}\left\{\left|\widehat{g}_{n}^{T} A \Pi^{(1)} W^{T} e_{n+1}\right|^{2}\right\}, \\
& V_{2} \triangleq \mathbb{E}\left\{\left|e_{n}^{T} A \Pi^{(1)} W^{T} \widehat{g}_{n+1}\right|^{2}\right\}, \\
& V_{3} \triangleq \mathbb{E}\left\{\left|e_{n}^{T} A \Pi^{(1)} W^{T} e_{n+1}\right|^{2}\right\} .
\end{aligned}
$$


The term $V_{1}$ can be calculated as

$$
\begin{aligned}
V_{1} & =\sigma_{e, n+1}^{2} \mathbb{E}\left\{\widehat{g}_{n}^{T} A \Pi^{(1)} W^{T 2}\right\} \\
& =\sigma_{e, n+1}^{2} \mathbb{E}\left\{\left[\left(\widehat{G}^{H} \widehat{G}\right)^{-1}\right]_{n+1, n+1}\right\}=\frac{\sigma_{e, n+1}^{2}}{(M-N) \sigma_{n+1}^{2}} .
\end{aligned}
$$

Similarly, we obtain $V_{2}=\left(\sigma_{e, n}^{2} /(M-K) \sigma_{n}^{2}\right)$, and $V_{3}=\left(\sigma_{e, n}^{2} \sigma_{e, n+1}^{2} / M(M-N)\right) \varrho$. Therefore,

$$
\operatorname{Var}\left(g_{n}^{T} b_{n+1}^{(1)}\right)=I_{n, n+1}
$$

(b) Compute $\mathbb{E}\left\{\left|g_{n}^{T} b_{n}^{(1)}\right|^{2}\right\}$, we get

$$
\mathbb{E}\left\{\left|g_{n}^{T} b_{n}^{(1)}\right|^{2}\right\}=\mathbb{E}\left\{\left|I_{1}+I_{2}+I_{3}\right|^{2}\right\} .
$$

Where $\mathrm{I}_{1} \triangleq \widehat{g}_{n}^{T} A \Pi^{(1)} W^{T} e_{n}, I_{2} \triangleq \widehat{e}_{n}^{T} A \Pi^{(1)} W^{T} \widehat{g}_{n}$, and $I_{3} \triangleq \widehat{e}_{n}^{T} A \Pi^{(1)} W^{T} e_{n}$. We obtain

$$
\mathbb{E}\left\{\left|g_{n}^{T} b_{n}^{(1)}\right|^{2}\right\}=\mathbb{E}\left\{\left|I_{1}\right|^{2}\right\}+\mathbb{E}\left\{\left|I_{2}\right|^{2}\right\}+\mathbb{E}\left\{\left|I_{3}\right|^{2}\right\} .
$$

Similarly, we have

$$
\begin{aligned}
& \mathbb{E}\left\{\left|I_{1}\right|^{2}\right\}=\frac{\beta_{n}-\sigma_{n}^{2}}{(M-K) \sigma_{n+1}^{2}}, \\
& \mathbb{E}\left\{\left|I_{2}\right|^{2}\right\}=\frac{\beta_{n}-\sigma_{n}^{2}}{(M-N) \sigma_{n-1}^{2}} .
\end{aligned}
$$

Next, we compute $\mathbb{E}\left\{\left|I_{3}\right|^{2}\right\}$. We obtain

$$
\begin{aligned}
\mathbb{E}\left\{\left|I_{3}\right|^{2}\right\} & =\sigma_{e, k}^{4} \mathbb{E}\left\{\operatorname{Tr}\left[\Pi^{(1)} \widehat{G}^{H} \widehat{G}^{*}\right)^{-1}\left(\Pi^{(1)}\right)^{H}\left(\widehat{G}^{T} \widehat{G}^{*}\right)^{-1}\right\} \\
& +\sigma_{e, k}^{4} \mathbb{E}\left\{\operatorname{Tr}\left[\Pi^{(1)} \widehat{G}^{H} \widehat{G}^{*}\right)^{-1}\left(\Pi^{(1)}\right)^{H}\left(\widehat{G}^{T} \widehat{G}^{*}\right)^{-1}\right\} \\
& \longrightarrow \frac{\sigma_{e, n}^{4} \varrho}{M(M-N)} .
\end{aligned}
$$

Substituting (A.15) and (A.16) into (A.13), we get

$$
\mathbb{E}\left\{\left|g_{n}^{T} b_{n}^{(1)}\right|^{2}\right\} \longrightarrow I_{n, n}
$$

(c) Compute $\mathbb{E}\left\{\left|g_{n}^{T} b_{n}^{(1)}\right|^{2}\right\}$, we obtain

$$
\mathbb{E}\left\{\left|g_{n}^{T} b_{n}^{(1)}\right|^{2}\right\} \longrightarrow I_{n, i}
$$

(d) Compute $\mathbb{E}\left\{\left\|g_{n}^{T} C^{(1)}\right\|^{2}\right\}$, we obtain

$$
\mathbb{E}\left\{\left\|g_{n}^{T} C^{(1)}\right\|^{2}\right\}=\frac{M+\sigma_{n+1}^{2} \sigma_{e, n}^{2} \varrho}{M(M-N) \sigma_{n+1}^{2}} .
$$

Substituting (A.11), (A.16), (A.18), and (A.19) into (A.7) yields (23).

\section{Data Availability}

The (software code) data used to support the findings of this study are available from the corresponding author upon request.

\section{Conflicts of Interest}

The authors declare that there are no conflicts of interest.

\section{Acknowledgments}

This work was supported in part by Key Scientific and Technological Projects in Henan Province, China (no. 192102210123), Key Research Fund for University in Henan Province, China (no. 20A520015), and Programs for Science and Technology Development in Henan Province, China (no. 212102210092).

\section{References}

[1] T. L. Marzetta, "Massive MIMO: an introduction," Bell Labs Technical Journal, vol. 20, pp. 11-22, 2015.

[2] X. Li, Q. Wang, Y. Liu, T. A. Tsiftsis, Z. Ding, and A. Nallanathan, "UAV-aided multi-way NOMA networks with residual hardware impairments," IEEE Wireless Communications Letters, vol. 9, 2020.

[3] T. L. Marzetta, "Noncooperative cellular wireless with unlimited numbers of base station antennas," IEEE Transactions on Wireless Communications, vol. 9, no. 11, pp. 3590-3600, 2010.

[4] C. D. Ho, "How to scale up the spectral efficiency of multi-way massive MIMO relaying?" IEEE International Conference on Communications IEEE, vol. 9, 2018.

[5] E. G. Larsson, O. Edfors, F. Tufvesson, and T. L. Marzetta, "Massive MIMO for next generation wireless systems," IEEE Communications Magazine, vol. 52, no. 2, pp. 186-195, 2014.

[6] X. Li, L. Li, F. Wen, J. Wang, and C. Deng, "Sum rate analysis of MU-MIMO with a 3D MIMO base station exploiting elevation features," International Journal of Antennas and Propagation, vol. 2015, p. 9, Article ID 318123, 2015.

[7] E. Björnson, E. G. Larsson, and T. L. Marzetta, "Massive MIMO: ten myths and one critical question," IEEE Communication, vol. 54, no. 2, pp. 114-123, 2016.

[8] G. Amarasuriya, C. Tellambura, and M. Ardakani, "Multi-way MIMO amplify-and-forward relay networks with zero-forcing transmission," IEEE Transactions on Communications, vol. 61, no. 12, pp. 4847-4863, 2013.

[9] C. D. Ho, H. Q. Ngo, M. Matthaiou, and T. Q. Duong, "On the performance of zero-forcing processing in multi-way massive MIMO relay networks," IEEE Communications Letters, vol. 21, no. 4, pp. 849-852, 2017.

[10] S. Silva, G. A. A. Baduge, M. Ardakani, and C. Tellambura, "NOMA-aided multi-way massive MIMO relay networks," IEEE Transactions on Communications, vol. 68, no. 7, pp. 4050-4062, 2019.

[11] G. Amarasuriya and H. V. Poor, "Multi-way amplify-and-forward relay networks with massive MIMO," 2015.

[12] G. Amarasuriya, E. G. Larsson, and H. V. Poor, "Wireless information and power transfer in multiway massive MIMO relay networks," IEEE Transactions on Wireless Communications, vol. 15, no. 6, pp. 3837-3855, 2016. 
[13] C. D. Ho, H. Q. Ngo, M. Matthaiou, and T. Q. Duong, "Multiway massive MIMO relay networks with maximum-ratio processing," in Proceedings of the 2017 International Conference on Recent Advances in Signal Processing, Telecommunications \& Computing (SigTelCom), IEEE, Da Nang, Vietnam, February 2017.

[14] G. Amarasuriya, C. Tellambura, and M. Ardakani, "Performance analysis of pairwise amplify-and-forward multi-way relay networks," IEEE Wireless Communications Letters, vol. 1, no. 5, pp. 524-527, 2012.

[15] G. Amarasuriya and H. V. Poor, "Impact of channel aging in multi-way relay networks with massive MIMO," IEEE Access, vol. 2015, 2015.

[16] F. Tan, "Energy-efficient power allocation for massive MIMO-enabled multi-way AF relay networks with channel aging," EURASIP Journal on Wireless Communications and Networking, vol. 9, 2018.

[17] S. Silva, G. A. A. Baduge, M. Ardakani, and C. Tellambura, "NOMA-aided multi-way massive MIMO relaying," IEEE Transactions on Communications, vol. 68, no. 7, pp. 40504062, 2020.

[18] F. Tan, T. Lv, and P. Huang, "Global energy efficiency optimization for wireless-powered massive MIMO aided multiway AF relay networks," IEEE Transactions on Signal Processing, vol. 66, no. 9, pp. 2384-2398, 2018.

[19] Q. Zhang, S. Jin, K.-K. Wong, H. Zhu, and M. Matthaiou, "Power scaling of uplink massive MIMO systems with arbitrary-rank channel means," IEEE Journal of Selected Topics in Signal Processing, vol. 8, no. 5, pp. 966-981, 2014.

[20] X. Li, J. Li, L. Li, L. Du, J. Jin, and D. Zhang, "Performance analysis of cooperative small cell systems under correlated Rician/Gamma fading channels," IET Signal Processing, vol. 12, no. 1, pp. 64-73, 2018.

[21] X. Li, M. Matthaiou, Y. Liu et al., "Multi-pair two-way massive MIMO relaying with hardware impairments over rician fading channels," IEEE Global Communication Conference, vol. 9, no. 13, pp. 1-6, 2018.

[22] C. D. Ho, "Multi-way massive MIMO with maximum-ratio processing and imperfect CSI," 2016.

[23] H. Q. Ngo, E. G. Larsson, and T. L. Marzetta, "Energy and spectral efficiency of very large multiuser MIMO systems," IEEE Transactions on Communications, vol. 61, no. 4, pp. 1436-1449, 2013.

[24] H. Q. Ngo, H. A. Suraweera, M. Matthaiou, and E. G. Larsson, "Multipair full-duplex relaying with massive arrays and linear processing," IEEE Journal on Selected Areas in Communications, vol. 32, no. 9, pp. 1721-1737, 2014. 